\title{
Left Atrial Size and Function After Spontaneous Cardioversion of Atrial Fibrillation and Their Relation to N-Terminal Atrial Natriuretic Peptide
}

\author{
Anna Vittoria Mattioli, MD, Silvia Bonatti, MD, Lorenzo Bonetti, MD, Paola Borella, MD, \\ and Giorgio Mattioli, MD
}

L eft atrial (LA) stunning after cardioversion of atrial fibrillation (AF) has been reported during spontaneous conversion to sinus rhythm. ${ }^{1}$ This observation suggest that atrial stunning is a function of underlying arrhythmia and not of the mode of cardioversion. It is known that $\mathrm{AF}$ causes atrial dilation, and progressive LA enlargement occurs when AF becomes chronic. ${ }^{2}$ Recently, it has been shown that multiple factors contribute to LA enlargement, including the presence and persistence of arrhythmia. ${ }^{3}$ Many reports suggest that if sinus rhythm is restored then dilation may regress. ${ }^{4}$ The Framingham Study showed a relation between LA size and the risk of stroke in men and the risk of death in both genders. ${ }^{5-7}$ Previous studies have suggested that $\mathrm{N}$-terminal atrial natriuretic peptide $(\mathrm{N}$ ANP) levels are elevated in patients with AF. ${ }^{8,9}$ It is unclear whether AF rather than LA dilation, ${ }^{10}$ hemodynamic impairment, ${ }^{11}$ or another hormonal alteration, can result in the elevation of N-ANP levels. ${ }^{8}$ The present report evaluates the changes in LA size and function after spontaneous cardioversion of $\mathrm{AF}$ and their relation to N-ANP.

Hemodynamically stable patients referred for cardioversion for nonrheumatic AF between September 1997 and March 2000 were considered for inclusion in this investigation. The initial study group included 202 consecutive patients; 98 patients spontaneously recovered sinus rhythm within 48 hours from the onset of arrhythmia and were selected for the study (Group A). The study population included 57 men and 41 women of mean age $60 \pm 16$ years; patients were compared with 98 age- and gender-matched control subjects (mean age $61 \pm 16$ years) who underwent pharmacologic cardioversion within 48 hours from the onset of arrhythmia (Group B). Patients received intravenous propafenone 2 $\mathrm{mg} / \mathrm{kg}$ of body weight; the drug was dissolved in $100 \mathrm{ml}$ of 5\% glucose and infused over 30 minutes. Exclusion criteria were: atrial flutter, valvular stenosis, valvular prosthesis, significant valvular insufficiency, atrial and/or left ventricular thrombosis, spontaneous echo contrast, patent foramen ovale or an atrial septal aneurysm, or decreased LV function (ejection fraction $<45 \%$ ). No patients received long-term therapy with antiarrhythmic drugs. Demographic and clinical characteristics of the

\footnotetext{
From the Departments of Cardiology and Biomedics, University of Modena and Reggio Emilia, Modena, Italy. Dr. Mattioli's address is: Department of Cardiology, University of Modena, Via del pozzo, 71, 41100 Modena, Italy. E-mail: mattioli.annavittoria@ unimo.it. Manuscript received December 17, 2002; revised manuscript received and accepted March 3, 2003.
}

\begin{tabular}{|lcc|}
\hline \multicolumn{3}{|c|}{ TABLE 1 Demographics and Clinical Characteristics } \\
\hline & Group A & Group B \\
& $(\mathrm{n}=98)$ & $(\mathrm{n}=98)$ \\
\hline Age $(\mathrm{yrs})$ & $60 \pm 16$ & $61 \pm 16$ \\
Men/women & $57 / 41$ & $58 / 40$ \\
Height $(\mathrm{cm})$ & $169 \pm 13$ & $171 \pm 9$ \\
Weight $(\mathrm{kg})$ & $85 \pm 19$ & $87 \pm 20$ \\
Body mass index $\left(\mathrm{kg} / \mathrm{m}^{2}\right)$ & $26 \pm 3$ & $25 \pm 2$ \\
Mean ventricular rate $($ beats $/ \mathrm{min})$ & $98 \pm 24$ & $97 \pm 26$ \\
Systemic hypertension & $24(24 \%)$ & $29(29 \%)$ \\
Diabetes mellitus & $9(9 \%)$ & $10(10 \%)$ \\
Coronary artery disease & $3(3 \%)$ & $1(1 \%)$ \\
Mean duration of AF (h) & $27 \pm 10$ & $29 \pm 9$ \\
\hline
\end{tabular}

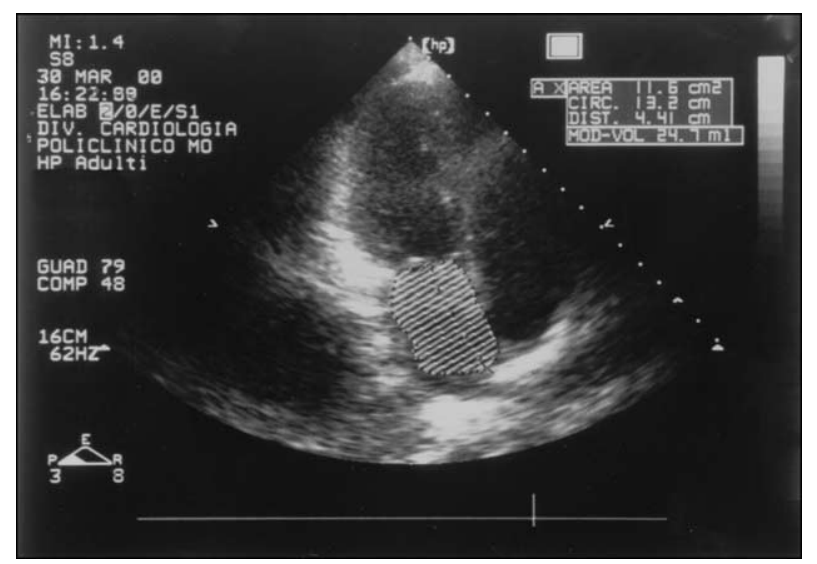

FIGURE 1. LA border traced in the apical 4-chamber view; LA volume calculated using Simpson's formula.

patients are listed in Table 1. Clinical records included age, gender, time and circumstances of the onset of symptoms related to $\mathrm{AF}$, and the duration of $\mathrm{AF}$ estimated from the initial onset of symptoms until the time of the in-hospital conversion. The protocol was approved by the Ethical Committee of our university and all patients signed an informed consent form.

The initial Doppler echocardiographic study was performed during AF and after cardioversion (mean 3 \pm 1.5 hours). A complete mono- and 2-dimensional color Doppler echocardiogram was performed in each patient using a commercial Hewlett-Packard echocardiograph (Andover, Massachusetts) with a $2.5-\mathrm{MHz}$ probe.

LA function was assessed using these parameters: (1) transmitral pulsed Doppler recorded from the apical 4-chamber view with the sample volume positioned between the tips of the mitral leaflets; peak early filling (E) and atrial filling (A) velocities; and 
TABLE 2 Serial Evaluation of Two-dimensional Echocardiographic Parameters Before and After Conversion to Sinus Rhythm in the Two Groups of Patients

\begin{tabular}{|c|c|c|c|c|}
\hline & $\begin{array}{l}\text { Group A Before } \\
\text { Spontaneous CV }\end{array}$ & $\begin{array}{l}\text { Group A After } \\
\text { Spontaneous CV }\end{array}$ & $\begin{array}{c}\text { Group B Before } \\
\text { Pharmacologic CV }\end{array}$ & $\begin{array}{c}\text { Group B After } \\
\text { Pharmacologic CV }\end{array}$ \\
\hline Heart rate (beats/min) & $98 \pm 24$ & $78 \pm 18 *$ & $97 \pm 26$ & $69 \pm 20 *$ \\
\hline Systolic blood pressure $(\mathrm{mm} \mathrm{Hg})$ & $134 \pm 22$ & $129 \pm 12$ & $138 \pm 24$ & $130 \pm 20$ \\
\hline Diastolic blood pressure (mm Hg) & $90 \pm 10$ & $87 \pm 11$ & $89 \pm 13$ & $88 \pm 9$ \\
\hline ANP (ng/ml) & $130 \pm 39$ & $47 \pm 29 *$ & $122 \pm 15$ & $51 \pm 23^{*}$ \\
\hline LA antero-posterior diameter $(\mathrm{mm})$ & $39 \pm 7$ & $34 \pm 4$ * & $41 \pm 3$ & $39 \pm 6$ * \\
\hline LA supero-inferior diameter $(\mathrm{mm})$ & $42 \pm 6$ & $38 \pm 4^{*}$ & $44 \pm 5$ & $40 \pm 6^{*}$ \\
\hline Maximal volume $\left(\mathrm{cm}^{3}\right)$ & $30 \pm 4$ & $27 \pm 6^{*}$ & $35 \pm 5$ & $30 \pm 6^{*}$ \\
\hline $\mathrm{P}$ atrial volume $\left(\mathrm{cm}^{3}\right)$ & $14 \pm 3$ & $12 \pm 5^{*}$ & $18 \pm 5$ & $14 \pm 6^{*}$ \\
\hline Minimal volume $\left(\mathrm{cm}^{3}\right)$ & $11 \pm 1.5$ & $9 \pm 3.1$ & $14 \pm 2$ & $10 \pm 4$ \\
\hline Conduit volume $\left(\mathrm{cm}^{3}\right)$ & $25 \pm 4$ & $26 \pm 5$ & $34 \pm 8$ & $33 \pm 6$ \\
\hline Passive emptying volume $\left(\mathrm{cm}^{3}\right)$ & $9 \pm 3.2$ & $8 \pm 2.8$ & $11 \pm 3.1$ & $10 \pm 3.3$ \\
\hline Active emptying volume $\left(\mathrm{cm}^{3}\right)$ & $6 \pm 2.4$ & $7 \pm 3.1$ & $7 \pm 2.1$ & $6 \pm 2$ \\
\hline
\end{tabular}

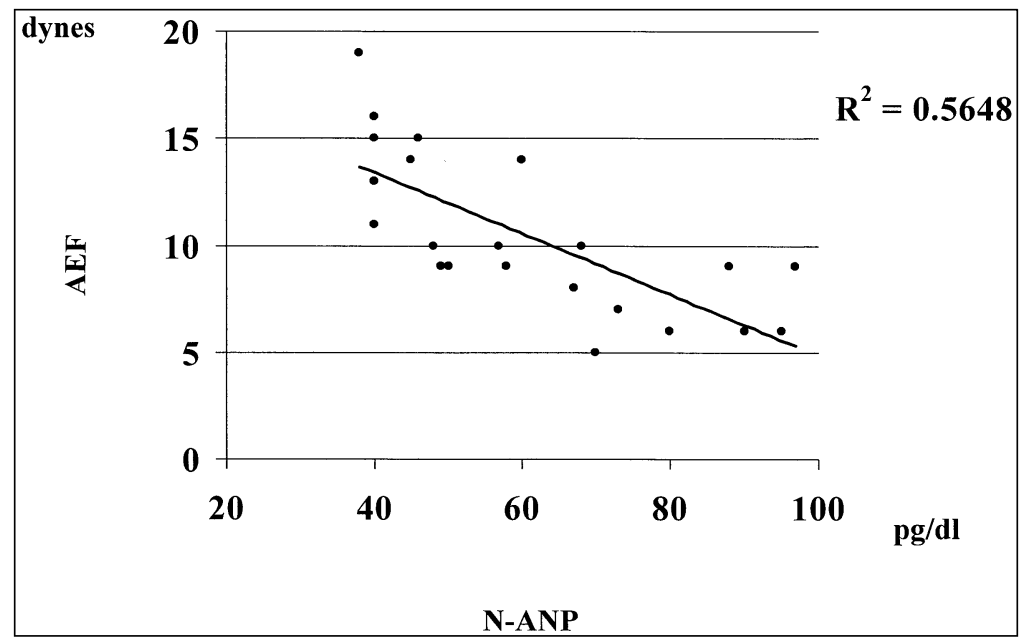

FIGURE 2. Correlation between atrial ejection force (FEA) and N-ANP levels (Anp) in patients with spontaneous conversion to sinus rhythm.

deceleration time and pressure halftime measured from the transmitral flow pattern. (2) Atrial ejection force was evaluated using this equation: $0.5 \times \rho \times$ mitral orifice area $\times(\text { peak } A \text { velocity })^{2}{ }^{12}$ Mitral orifice area was assumed to be circular and was estimated from the mitral annulus diameter as measured from the apical 4-chamber view.

LA size was measured during systole along the parasternal long-axis view from 2-dimensionally guided M-mode tracing. ${ }^{13}$ LA volumes were determined at the mitral valve opening (maximal volume), at the onset of atrial systole ( $\mathrm{P}$ wave of electrocardiogram, $\mathrm{P}$ volume), and at mitral valve closure (minimal volume). LA volumes were measured from the apical 4- and 2-chamber views by means of the biplane area-length method, and corrected for body surface area (Figure 1). ${ }^{14}$ These parameters were estimated from the LA volumes: LA passive emptying was assessed as LA passive emptying volume (maximal volume - $\mathrm{P}$ volume), conduit volume (left ventricular stroke volume - [maximal volume - minimal volume]), and LA passive emptying fraction (LA passive emptying volume/maximal volume); LA active emptying was estimated using LA active emptying volume ( $\mathrm{P}$ volume - minimal volume) and LA active emptying fraction (LA active emptying volume/P volume); LA total emptying volume (maximal volume - minimal volume); contribution of passive emptying volume ([maximal volume - $\mathrm{P}$ volume]/stroke volume), of conduit volume (conduit volume/ stroke volume), and of active emptying volume ([P volume - minimal volume]/stroke volume) to left ventricular stroke volume. ${ }^{15}$

Patients with moderate and severe mitral regurgitation were excluded from the study. All Doppler results are mean measurements of 5 cardiac cycles. The Doppler signal was analyzed using an IBM computer (Milan,

Italy).

Blood samples were collected in tubes that contained ethylenediamine tetraacetic acid $(7.5 \mathrm{mmol})$ and a protease inhibitor (aprotinin). The plasma was separated by centrifugation $\left(2,500 \mathrm{rpm}\right.$ at $\left.4^{\circ} \mathrm{C}\right)$ and kept at $-35^{\circ} \mathrm{C}$ until the determination of N-ANP concentration by a radioimmunoassay technique. The sensitivity of the method in our laboratory was 3 $\mathrm{pg} / \mathrm{ml}$, and inter- and intra-assay coefficients of variation were $16 \%$ and $14 \%$, respectively. ${ }^{16}$

Data are expressed as mean $\pm 1 \mathrm{SD}$ for continuous variables. The statistical significance of serial changes in echocardiographic parameters was determined by the analysis of variance for repeated measures. Pearson's correlation coefficient was used to study the correlation between 2 parameters. The predictive value of clinical and echocardiographic indexes for spontaneous conversion was assessed with a logistic regression analysis. The dependent variable was spontaneous conversion to sinus rhythm; covariates included age, gender, time of onset of $\mathrm{AF}$, duration of arrhythmia, LA volumes, N-ANP, and atrial function. 


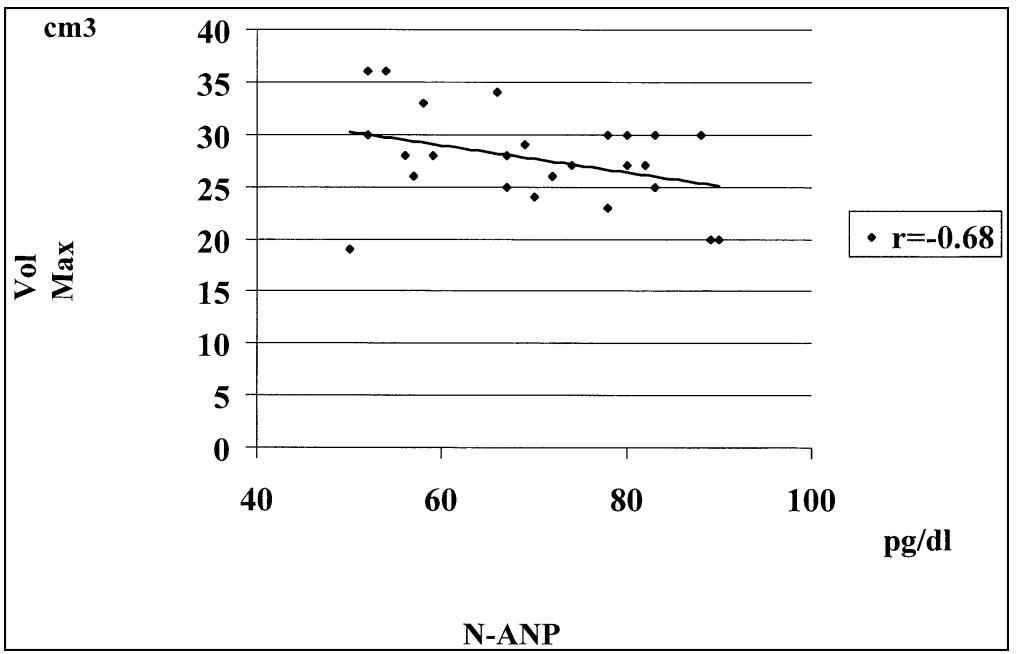

FIGURE 3. Correlation between LA maximal volume and N-ANP levels (ANP) in patients with spontaneous conversion to sinus rhythm.

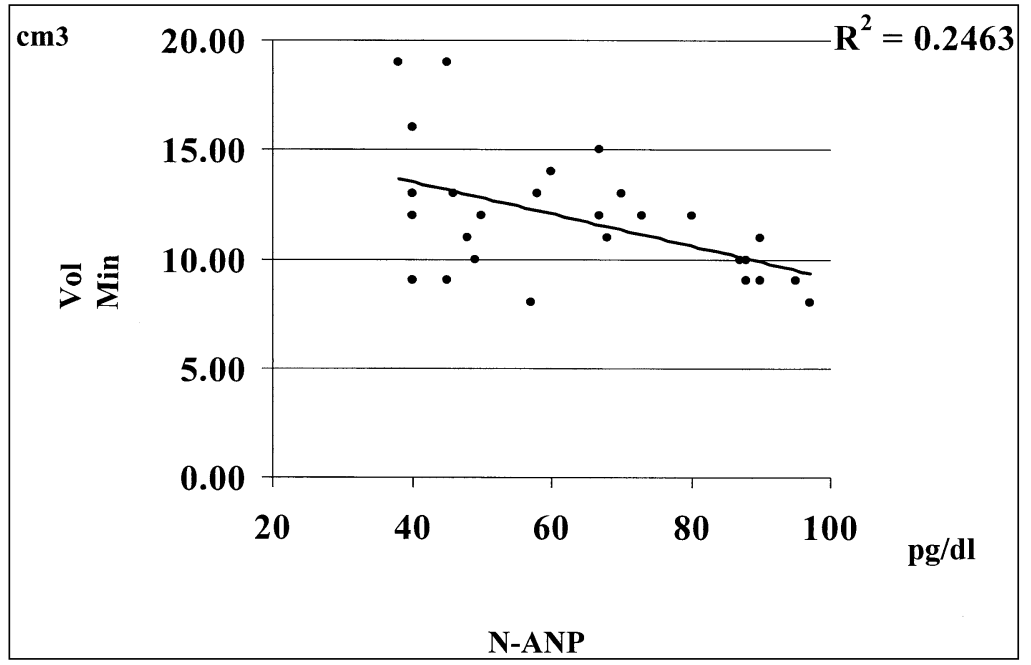

FIGURE 4. Correlation between LA minimal volume (Vol Min) and N-ANP levels (Anp) in patients with spontaneous conversion to sinus rhythm.

was reported between atrial ejection force and LA diameter $(r=-0.67, p$ $<0.001$ in Group A and $r=-0.56, p$ $<0.01$ in Group B). Patients with a lower atrial ejection force had a larger left atrium persisting after the recovery of sinus rhythm.

The left atrium was enlarged in all patients during arrhythmia. LA volumes were greater during $\mathrm{AF}$ compared with volumes recorded after the restoration of sinus rhythm. Serial evaluation of LA diameters and volumes are listed in Table 2. N-ANP was measured before and immediately after cardioversion. A comparison of hormonal levels between groups showed that patients with spontaneous conversion had significantly higher N-ANP levels than patients of Group B during AF (312 \pm 98 vs $245 \pm 87 \mathrm{pg} / \mathrm{dl} ; \mathrm{p}<0.001)$. A comparison between groups in sinus rhythm showed higher N-ANP levels in patients with spontaneous conversion even if the value was not significant $(189 \pm 48$ vs $145 \pm 54 \mathrm{pg} / \mathrm{dl} ; \mathrm{p}$ $=\mathrm{NS})$. A significant correlation was found between atrial ejection force and N-ANP level $(\mathrm{r}=-0.73, \mathrm{p}$ $<0.001$; Figure 2). A significant inverse correlation was observed between LA maximal volume and $\mathrm{N}$ ANP level $(r=-0.68 ; p<0.0001)$ in patients with spontaneous conversion to sinus rhythm, whereas the relation with LA minimal volume was weak ( $\mathrm{r}$ $=-0.41, p<0.01$; Figures 3 and 4$)$.

Logistic regression analysis permitted the identification of factors influencing spontaneous conversion to sinus rhythm. No significant differ-

Odds ratios and $95 \%$ confidence intervals were calculated based on results of the logistic regression analysis. A $p$ value $<0.05$ was considered significant.

Mean duration of AF was $27 \pm 10$ hours in Group A and $29 \pm 9$ hours in Group B $(p=N S)$. Mean heart rate was higher in patients during $\mathrm{AF}$ compared with that evaluated after conversion in the 2 groups of patients. Systolic and diastolic blood pressures did not change after conversion to sinus rhythm in either group (Table 2). All patients had an adequate echocardiogram.

The recovery of atrial mechanical function was measured using peak A wave velocity and the atrial ejection force. Atrial ejection force decreased in 56 patients after spontaneous restoration of sinus rhythm (8.2 \pm 3 dynes) and was normal in the other patients (11 \pm 3 dynes); it slightly decreased after pharmacologic cardioversion ( $7 \pm 2$ dynes). Serial evaluation of atrial ejection force values and of peak $\mathrm{A}$ wave velocities are listed in Table 2. An inverse correlation ences were observed concerning age, gender, or duration of AF. Logistic regression analysis of echocardiographic data was used to identify predictors of spontaneous conversion to sinus rhythm. The most important predictor of spontaneous conversion was the plasma concentration of N-ANP during the arrhythmia; patients with levels of N-ANP over the median value experienced spontaneous conversion (odds ratio 3.20, 95\% confidence interval 1 to 4.12 ). Others predictors of spontaneous conversion were a maximal volume of the left atrium $<30 \mathrm{~mm}^{3}$ (odds ratio $2.1,95 \%$ confidence interval 1.1 to 3.0 ) and a minimal volume $<12 \mathrm{~mm}^{3}$ (odds ratio $1.5,95 \%$ confidence interval 0.9 to 4.0 ).

The present investigation shows that recovery of atrial mechanical function was delayed in patients with spontaneous conversion of AF. N-ANP levels increased in patients with AF that spontaneously developed sinus rhythm compared with patients who did 
TABLE 3 Correlation Between Echocardiographic Parameters and N-terminal Atrial Natriuretic Peptide (N-ANP)

\begin{tabular}{|lll|}
\hline & Group A & Group B \\
\hline LA antero-posterior diameter & $r=-0.26$ & $r=-0.48$ \\
& $p=N S$ & $p<0.05$ \\
LA supero-inferior diameter & $r=-0.26$ & $r=-0.48$ \\
LA maximal volume & $p=N S$ & $p<0.05$ \\
& $r=-0.68$ & $r=-0.40$ \\
LA minimal volume & $p<0.0001$ & $p<0.05$ \\
Left ventricular end-diastolic diameter & $p=0.01$ & $p=N S$ \\
& $p=N S$ & $r=0.29$ \\
Left ventricular end-systolic diameter & $r=0.28$ & $r=0.51$ \\
& $p=N S$ & $p<0.05$ \\
Left ventricular ejection fraction & $r=0.34$ & $r=0.32$ \\
& $p=N S$ & $p=N S$ \\
\hline
\end{tabular}

not spontaneously develop sinus rhythm. Spontaneous conversion to sinus rhythm was associated with higher values of N-ANP during AF and with smaller LA volumes. This study evaluated the modification of LA diameters and volumes during AF and after spontaneous conversion to sinus rhythm. Patients were compared with a control group of subjects who underwent successful pharmacologic cardioversion. We selected patients who spontaneously reverted to sinus rhythm to exclude interference on LA size due to atrial stunning after cardioversion. In our experience, the recovery of atrial mechanical function occurs promptly after spontaneous conversion of AF. ${ }^{16,17}$ Mean duration of $\mathrm{AF}$ in patients from our investigation was $<36$ hours, suggesting that an early cardioversion of arrhythmia can prevent atrial stunning. Nevertheless, a study by Rubin and colleagues ${ }^{18}$ suggested that changes in the LA anatomy and function occur quite soon after the initiation of AF. Histologic changes are associated with anatomic macroscopic changes (i.e., LA dilation and changes in atrial volume and shape). ${ }^{13}$ The shape of the left atrium is believed to be modified during arrhythmia due to the loss of organized contraction. These changes occurring in the atria during AF may have a deleterious effect on the atria when sinus rhythm is restored. The decrease of N-ANP levels observed after cardioversion does not exclude the possibility of atrial myocite alteration and may reflect the transient inhibition of N-ANP secretion. ${ }^{8,19}$

The decrease of the supero-inferior diameter was greater compared with decreases of the antero-posterior and medio-lateral diameters. This feature suggests a hemodynamic change in the left and right atrium due to an increased mean right atrial pressure to maintain an adequate left ventricular filling.
In conclusion, higher levels of $\mathrm{N}$-ANP during AF were independently associated with spontaneous conversion, as well as with smaller LA volume. An inverse correlation existed between LA volume and N-ANP.

1. Khan IA. Transient atrial mechanical dysfunction (stunning) after cardioversion of atrial fibrillation and flutter. Am Heart J 2002;144:11-22.

2. Sanfilippo AJ, Abascal VM, Sheehan M, Oertel LB, Harrigan P, Hughes RA, Weyman AE. Atrial enlargment as a consequence of atrial fibrillation. A prospective echocardiographic study. Circulation 1990;82:792-797.

3. Dittrich HC, Pearce LA, Asinger RW, McBride R, Webel R, Zabalgoitia M, Pennock GD, Safford RE, Rothbart RM, Halperin JL, Hart RG, on behalf of the Stroke Prevention in Atrial Fibrillation Investigators. Left atrial diameter in nonvalvular atrial fibrillation: an echocardiographic study. Am Heart J 1999; 137: 494-499.

4. Welikovitch L, Lafreniere G, Burggraf GW, Sanfilippo AJ. Change in atrial volume following restoration of sinus rhythm in patients with atrial fibrillation: a prospective echocardiographic study. Can J Cardiol 1994;10:993-996.

5. The Stroke Prevention in Atrial Fibrillation Investigators. Predictors of thromboembolism in atrial fibrillation. Echocardiographic features of patients at risk. Ann Intern Med 1992;116:6-12.

6. Benjamin EJ, D'Agostino RB, Belanger AJ, Wolf PA, Levy D. Left atrial size and the risk of stroke and death. The Framingham Heart Study. Circulation 1995;92:835-841.

7. Caplan LR, D'Cruz I, Hier DB, Reddy H, Shah S. Atrial size, atrial fibrillation and stroke. Ann Neurol 1986;19:158-161.

8. Rossi A, Enriquez-Sarano M, Burnett JC Jr, Lerman A, Abel MD, Seward JB. Natriuretic peptide levels in atrial fibrillation. A prospective hormonal and Doppler-echocardiographic study. J Am Coll Cardiol 2000;35:1256-1262.

9. Berglund H, Boukter S, Theodorsson E, Vallin H, Edhag O. Raised plasma concentrations of atrial natriuretic peptide are independent of left atrial dimensions in patients with chronic atrial fibrillation. Br Heart J 1990;64:9-13.

10. Globits S, Frank H, Pacher B, Huelsmann M, Ogris E, Pacher R. Atrial natriuretic peptide release is more dependent on atrial filling volume than on filling pressure in chronic congestive heart failure. Am Heart J 1998;135:592597.

11. Hornestam B, Hall C, Held P, Carlsson T, Falk L, Karlson BW, Lundstrom T, Peterson M. N-terminal proANF in acute atrial fibrillation: a biochemical marker of atrial pressures but not a predictor for conversion to sinus rhythm. Am Heart J 1998;135:1040-1047.

12. Manning WJ, Silverman M, Katz SE, Douglas PS. Atrial ejection force. A noninvasive assessment of atrial systolic function. J Am Coll Cardiol 1993;22: 221-225.

13. Sahn DJ, DeMaria A, Kisslo J, Weiman A. Recommendations regarding quantitation in M-mode echocardiography: results of a survey of echocardiographic measurements. Circulation 1978;58:1072-1083.

14. Mattioli AV, Sansoni S, Ricci Lucchi G, Mattioli G. Serial evaluation of left atrial dimension after cardioversion for atrial fibrillation and relation to atrial function. Am J Cardiol 2000;85:832-836.

15. Toutouzas K, Trikas A, Pitsavos C, Barbetseas J, Androulakis A, Stefanadis C, Toutouzas P. Echocardiographic features of left atrium in elite male athletes. Am J Cardiol 1996;78:1314-1317.

16. Mattioli AV, Castelli A, Andria A, Mattioli G. Clinical and echocardiographic features influencing recovery of atrial function after cardioversion of atrial fibrillation. Am J Cardiol 1998;82:1368-1371.

17. Mattioli AV, Castelli A, Bastia E, Mattioli G. Atrial ejection force in patients with atrial fibrillation: comparison between DC shock and pharmacological cardioversion. Pacing Clin Electrophysiol 1999;22:33-38.

18. Rubin DN, Katz SE, Riley MF, Douglas PS, Manning WJ. Evaluation of left atrial appendage anatomy and function in recent-onset atrial fibrillation by transesophageal echocardiography. Am J Cardiol 1996;78:774-778.

19. Petersen P, Kastrup J, Vilhelmsen R, Schutten HJ. Atrial natriuretic peptide in atrial fibrillation before and after electrical cardioversion therapy. Eur Heart J 1988;9:639-641. 